\title{
PROTECTION AND CONTROL OF DC MICRO GRIDS USING RASPBERRY PI
}

\author{
S.C. Vijayakumar \\ Asst. Professor, Department of \\ Electrical and Electronics \\ Engineering, Vel \\ Tech,Chennai, India
}

\author{
S.Thanigaivel \\ U.G. Scholar, Department \\ of Electrical and \\ Electronics Engineering, \\ Vel Tech, Chennai, India,
}

\author{
R.Selvaganapathy \\ U.G. Scholar, Department \\ of Electrical and \\ Electronics Engineering, \\ Vel Tech, Chennai, India
}

\author{
R.Nishant kumar \\ U.G. Scholar, \\ Department of Electrical \\ and Electronics \\ Engineering, Vel Tech, \\ Chennai, India
}

\begin{abstract}
The major objective of these manuscripts is to build up a computerized framework dependent in idea of Internet of Things which continually screens the electrical parameters to be specific current as well as voltage coursing by different parts of DC Micro network, distinguishes as well as control the issue by segregating the lattice and reconnecting with another source. The client would be alarmed during event of different shortcomings. It will likewise distinguish the open circuit during substantial stormy condition and breaks the circuit to ensure the mayhem.
\end{abstract}

Keywords - Internet of Things, DC Micro Network, open circuit, computerized framework, current, voltage, lattice.

\section{INTRODUCTION}

Location of Faults is one of the major problems in power systems. It is very significant parameter for developing working effectiveness as well as unwavering power quality framework to distinguish the time taken ( $\mathrm{Li}$ et al., 2018; Majumder et al., 2017; Tu et al., 2018). In our nation we utilize a brought together Power matrix partitioned into 5 zones to control the whole nation (North, South, East, North East, and West). This makes the matrix exceptionally hardened and requests profoundly arranged and sorted out activities with respect to any progressions done in power framework to guarantee security as well as safe activity of National lattice. If the framework can be divided as littler segments as well as worked as a system of such littler pieces, the matrix may computerized and worked along greater proficiency (Mokhberdoran et al., 2017; Meghwani et al., 2015; Bakar et al., 2014). Development of DC small scale matrices implies the size as well as unpredictability of the framework to be constrained is decreased definitely. The idea of IoT will help computerize the power lattice as well as empower the power frameworks designer work as overseeing authority of a mechanized procedure.

Section II explains the suggested IOT based protection as well as control system to micro grids.

Section III illustrates system specifications.
Test cases of sample fault as well as its IOT based detection of fault is explained by section IV.

\section{A. Internet of Things}

Web innovation gives a decent method to us to build up the coordinated registering system condition. IoT denotes physical gadget system, vehicles, household apparatuses, other materials installed along hardware, scheduling sensors actuators as well as system network, which authorize such substance to meet as well as deal information. All is exceptionally identifiable by its entrenched process framework still may interoperate within the current web foundation. As per Experts the IoT would consists of for about 30 billion items by 2020 .

IoT helps things to be found or crosswise remotely control over previous system base (Peralta et al., 2019; Thakkar et al., 2015; Swathika et al., 2019). Creating more open doors to direct mix of physical world with PC depend on frameworks, bring improved productivity, precision as well as financial benefit notwithstanding decreased human intercession. In the point while IoT is increased to sensors as well as actuators, invention turns with case of much wide class of digital - a physical frameworks digital physical framework that likewise envelops advances, for instance, savvy lattices, virtual power plant, keen home, wise transport as well as shrewd urban areas (Gnana et al., 2016).

Things", at IoT, could implies to the broad collection of gadgets, for instance, heart checking inserts, biochip transponders at livestock, Using IoT we can see the cameras gushing exist feeds of wild creature at beach front waters, automobiles along implied sensors, DNA assessment gadgets to usual, sustenance, pathogen seen or field activity gadgets which enable firemen at inquiry as well as salvage tasks. Lawful researchers suggests with respect to "things" as "blend of equipment, scheduling, information as well as administration" (Swathika et al., 2016a; Swathika et al., 2016b).

Such gadgets collect expensive in sequence to help of various previous advancements then independently send the 
information among various gadgets. The progressive extension of Internet-associated items is additionally expected to create many information from various areas, along the resulting require to snappy accumulation of information, an expansion at require to record, store, procedure these information all the more successfully. As late with huge development in worldwide digital risk, there has been a critical ascent in abuse of IoT innovations for carrying out digital fear wrongdoings.

\section{B. Raspberry pi-3}

Raspberry pi 3 model denotes third generation. It depends upon wide com BCM2837 chip. SD card utilized to long expression storage as well as booting. 40 pins denotes these Raspberry pi 3 model pin diagram. It has a SD card slot which can help awake with 64 GB memory card. Raspberry pi has a Quad core processor. It consists of 64 bit as well as $1.2 \mathrm{GHz}$ clock speed (Approximately 50\% quicker to pi 2). Raspberry pi 3 consists of 1 GB RAM.

Small, powerful, lightweight as well as ARM based computer are the advantages of Raspberry Pi 3 model. It could do things which normal computer could do. Through Raspberry Pi Foundation it was developed on UK. It is developed to inspire the learners generation to creative as well as discover that how a computer is program as well as to know their function. This is separate Linux, BSD, RISC OS, or Plan 9 system along more I/O. The ability of powerful graphics as well as HDMI video output creates it perfect to multimedia utilizations like media centers as well as thin casting methods. Raspberry Pi denotes in terms of Broadcom BCM2837 chip. This do not has construct-at hard disk or solid-state drive; in its place it consists of SD card slot which can support SD card up to $64 \mathrm{~GB}$ to boot as well as long-term storage.

Raspberry pi doesn't have a worked on hard circle or strong state drive such as conventional PCs rather it has a SD card to firing up as well as putting away of data. Raspberry Pi SD card works just like a hard drive on a conventional PC. SD card should comprise working framework, programs, information expected to execute the Raspberry Pi. The working framework advises Raspberry Pi how to work, how to deal with any contribution from the client as well as how to oversee programs while they were running.

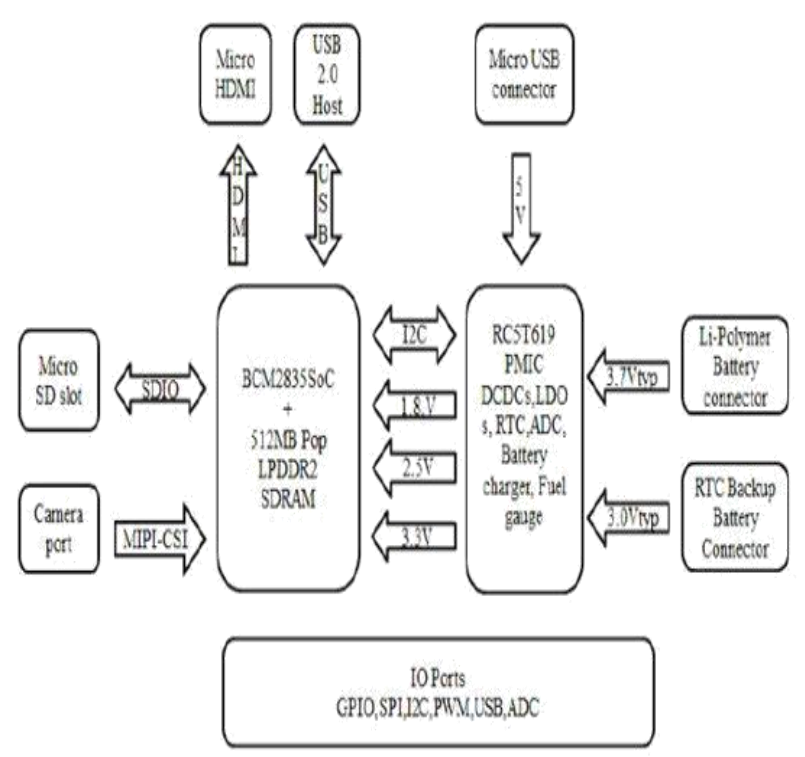

Fig.1. Raspberry pi-3 model Block Diagram

\section{Relay}

An electrically operated switch is called relay. To operate a switch several relays utilize an electromagnet, yet another operating principle denotes employed, like solid-state relay. These utilized here it is necessary with control a circuit through individual minimum-power signal, or here many circuits should control through a signal. Control a circuit through individual minimum-power signal, or here many circuits should control through a signal is the major application of the relays. It is also used to trip the circuit whenever fault occurs.

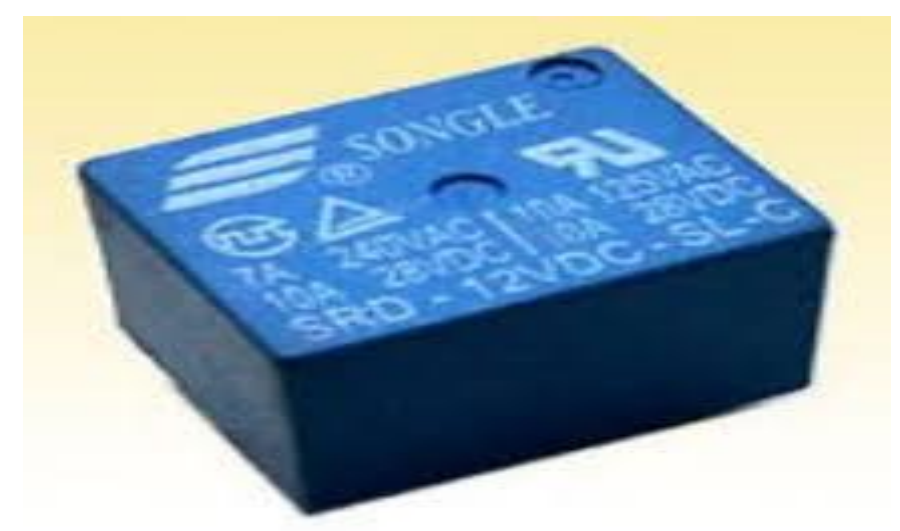

Fig.2. Relay

\section{MCP3008 Analog to Digital Converter}

Analog-to-digital converter (ADC, A/D, or A-to-D) denotes systems which convert the analog signal in to digital signal, such as sound is taken through microphone or else light entering the digital camera, into High and low format. ADC 


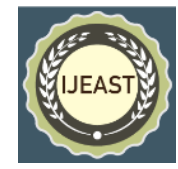

can offer a measurement like electronic device that convert input analog voltage or else current with digital number indicating voltage magnitude or current. Usually, digital results represent complementary binary number of the two that is proportionate with input, but there are other possibilities.

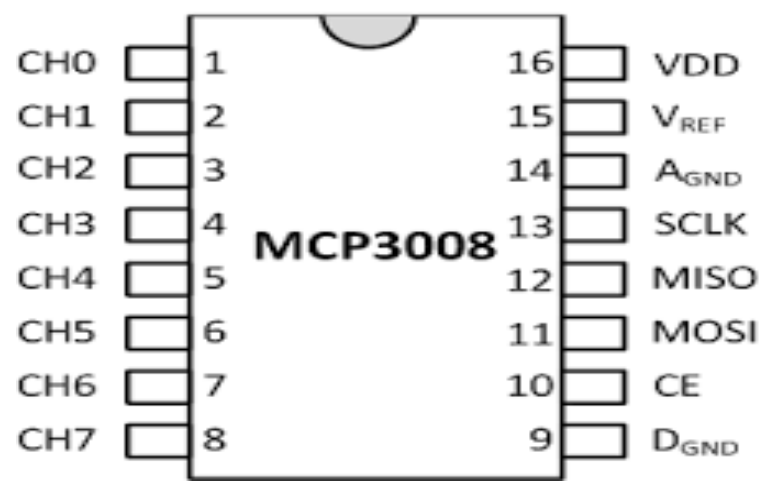

Fig.3. Pin Configuration of ADC (Mcp3008)

\section{E. Voltage Sensor}

A trimpot is a small potentiometer it is also called as trimmer potentiometer that is utilized to adjustment, tuning as well as calibration in circuits. During trimpots are utilized into changeable resistance (wired as rheostat) is known as predetermined resistors. Trimpots or else preset are generally mounted in written circuit boards as well as familiar through utilizing a screwdriver. Materials are utilized as resistive track denotes unreliable, yet very general is also carbon composition or else cermet. For occasional adjustment Trimpot is planned also can reach a higher resolution while utilizing multi-turn setting screws. During trimmer potentiometer is utilized into substitute to ordinary potentiometers, care must taken as their lifespan designed is often 200 cycles only.

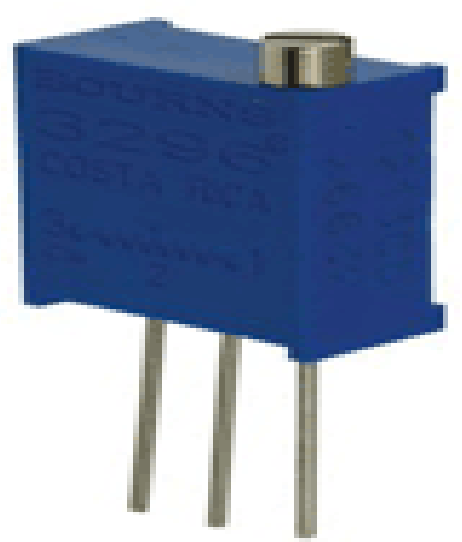

Fig.4. Trimmer pot

\section{F. Current Sensor}

Both open as well as closed loop type is current sensor. Both $\mathrm{AC}$ as well as DC currents is computed by open loop current sensor as well as gives electric insulation among the circuits. Open loop sensor is better than close loop sensor because open loop sensor is low expense. Generally open loop current sensor is preferred at battery operated devices.

Closed loop sensor is appropriate for commercial as well as industrial utilizations. They have high precision in environment as well as maximal temperature. They are good in noisy ambient as well as can easily turn their output into voltage.

LEM - LTS 25 - NP is one of the good examples for closed loop current sensors. It is more good efficiency, well linearity as well as much minimal temperature drift. Key features of LTS 25-NP:

- Unipolar voltage supply

- Insulated plastic box identified due to UL 94-V0

- Compact PCB mount design

- Incorporates resistance measure

- Comprehensive range measure

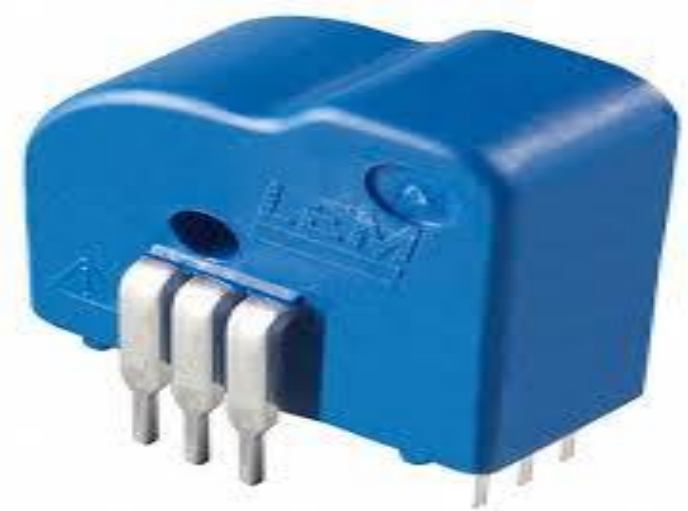

Fig.5. Current Sensor

\section{WORKING PRINCIPLE}

In this project we are sensing the current and voltage value from the grid using the current sensor and voltage sensor, then we are converting this into digital utilizing the converter of analog to digital (mcp3008) as well as providing this value for our Raspberry pi. In the micro controller we have some preset value for current and voltage by which we can check whether the voltage or current goes abnormal. If it happens then our micro controller will drive the relay and trips the circuit, then connects the load to a new source and notify us using a message and by alarming. Using our project we can detect any open circuit during natural disasters such as cutting of line wire during heavy rain and by detecting this we can break off the circuit.

\section{EXISTING Methodologies}

Directional defense depends on energy function of failure component at microgrid ( $\mathrm{Li}, \mathrm{Li}, \mathrm{Fu}$ and $\mathrm{Li}, 2012)$. They are using Directional protection which is complex and time consuming. In this we have to identify the fault type first. 
Grid -tied in VSI protection beside voltage-based grid side failures (Majumder, Roy and Chowdhuri, 2017). They are using voltage in terms of safety system which involves fault detection of grid side along automated islanding method of 3phase inverter following failure. Following isolating inverter, it would get mind of its possess local load as well as operate at isolated model till grid failure is vacant as well as VSI is reconnected with grid and supply the load.

Arm Overcurrent Protection as well as integration has been introduced in MMC-HVDC (Tu et al., 2018). In this method coordination of arm overcurrent a defensive behavior that is configured at valve controls as well as DC protection of modular multilevel converter depends on HVDC (MMCHVDC). Mismatch of parameter at valve control as well as DC protection can guide with unforeseen block, ac protection harmonization figure is utilized with examine the location as well as time delay of arm overcurrent protection. A provisional block function at valve control was suggested as well as it is configured properly along another protection levels at DC protection device.

HVDC grids protection utilizing unidirectional DC circuit breakers as well as rapid local protection algorithm was introduced (Mokhberdoran, Pirooz Azad, Van Hertem, Silva and Carvalho, 2017). The utilization of one-way DC circuit breakers, such as the current-release DC circuit breaker, was introduced at protection of multi-terminal HVDC grids. Moreover, new suggested rapid non-unit protection algorithm is used to unidirectional protection of multi terminal grid. This dissertation outcome proved which one-way DC circuit breakers may defend DC grids like bidirectional DC circuit breaker.

To DC micro grid, a new safety scheme utilizing line current derivative is introduced (Meghwani, Srivastava and Chakrabarti, 2015). This method is in terms of DC current profile concept under transients that based upon location of fault. This paper depends on directional feature to reach the DC micro grid protection. Suggested method is proved in ring type DC micro grid system that can to find the fault at DC system, also actuates its backup security.

\section{PROPOSED SYSTEM FOR MICRO GRIDS}

In this dissertation the suggested system automatically tracking the current as well as voltage at every micro grid as well as alerts the user while the faults of over current (Bakar et al., 2014), ground, short circuit. System will have the ability to control the appropriate safety circuits by eliminating critical faults instantly. The micro grid function is automated, eliminating require to human decision making as well as can greatly decrease the less reaction time required to respond for fault conditions (Peralta, Faicán and Pinos, 2019; Thakkar, 2015)

For realizing the suggested scheme, transducers should be established in appropriate locations on the micro grid buses in terms of operational ranges of transducers (Swathika and Hemamalini, 2019; Bedekar, Bhide and Kale, 2010). The data obtained through transducers is gathered through microcontroller, from transducers the signal of output is transferred to the desired logic state then uploaded to the cloud, for effective information in human understandable formats where raw data is processed (Gnana Swathika and Hemamalini, 2016; Swathika, Hemamalini, Garg and Aggarwal, 2016; Swathika, Hemamalini, Mishra, Pophali and Barve, 2016). By authorized users processed data could viewed remotely. During fault detection, system issues signals at form of SMS, e-mail as well as on-screen notifications to users. To instantly clear critical faults the system induces safe circuit actuators such as relays, isolators, circuit breakers.

System consists of ability to implement role denoted in Figure1 to reach the suggested functions:

- Obtaining data from transducers in suitable data transfer rates.

- Signal conditioning and noise reduction

- Changing signals to appropriate logic levels

- Data uploading to cloud utilizing correct hardware as well as communication protocols

- Trigger actuators on receiving commands from cloud

\section{A. Circuit Diagram and Block Diagram Of Proposed System}

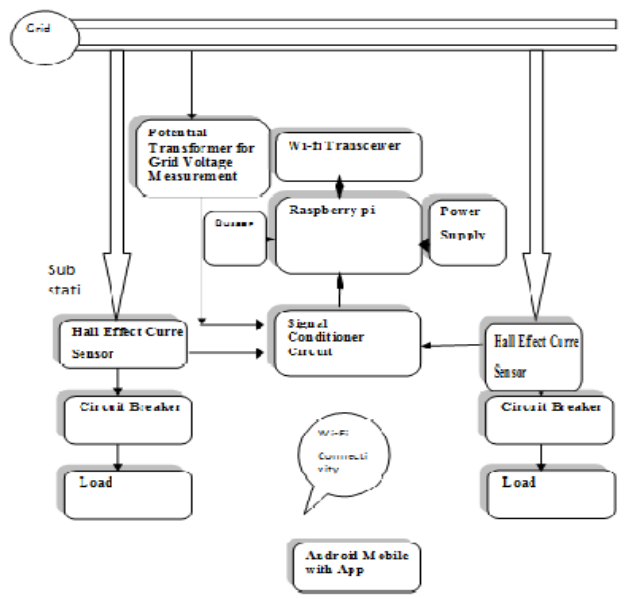

Fig. 6. Block Diagram of proposed System

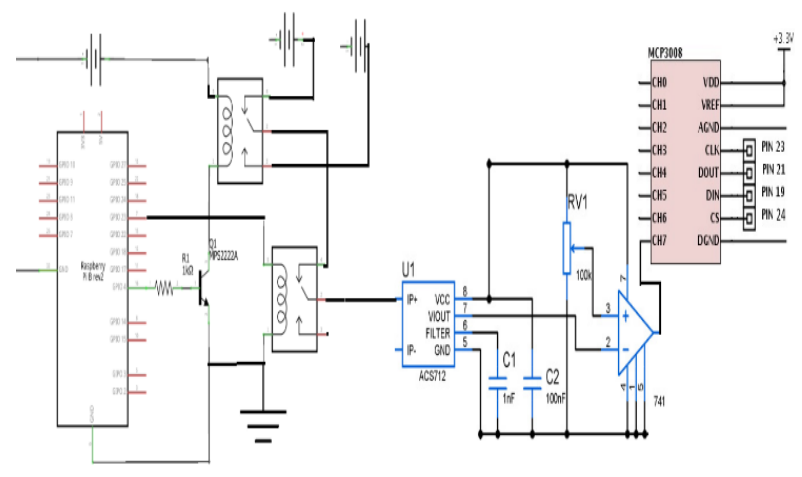


Fig.7. Circuit Diagram of Proposed System

\section{HARDWARE AND SOFTWARE DESCRIPTION}

$\begin{array}{ll}\text { Microcontroller } & \text { : Raspberry pi-3 } \\ \text { Internet module } & \text { : Raspberry Pi Ethernet Shield } \\ \text { Transducer } & \text { : LTS 25-NP Hall-Effect Current Sensor } \\ \text { Actuator } & \text { : SRD-05VDC-ELC-30A Relay } \\ \text { IoT Platform } & \text { : MobaXterm }\end{array}$

LEM - LTS 25 - NP is one of the good examples for closed loop current sensors. It has very good efficiency, good linearity and very low temperature drift.

Voltage input $: 5 \mathrm{~V} \mathrm{DC}$

Operating range : $-30 \mathrm{~A}$ to $+30 \mathrm{~A}$ max.

SRD-05VDC-SL-C Relay is utilized while actuator at the prototype. Relay may utilized to both $\mathrm{AC}$ as well as DC

Input signal $: 5 \mathrm{~V} \mathrm{DC}$

RASPBERRY PI 3 MODEL SPECIFICATIONS

SoC - Broadcom BCM2837 64bit ARMv8 quad core Cortex A53 processor@1.2GHz to double core Video Core IV GPU @ 400 $\mathrm{MHz}$ helps OpenGL ES 2.0, hardware-accelerated Open VG, $1080 \mathrm{p} 30$ H.264 great-profile decode. Able tolGpixel/s, $1.5 \mathrm{Gtexel} / \mathrm{s}$ or $24 \mathrm{GFLOPs}$ to surface filter as well as DMA infrastructure.

System Memory - 1GB LPDDR2.

Storage - micro SD slot.

Audio \& Video Output - HDMI 1.4 as well as 4-pole stereo audio as well as composite video port.

Connectivity - 10/100M Ethernet, Wi-Fi $802.11 \mathrm{~b} / \mathrm{g} / \mathrm{n}$ up to 150Mbps and Bluetooth 4.1 LE (BCM43438 module).

USB - 4x USB 2.0 host ports (to best power management, includes maximum power peripheral), $1 \mathrm{x}$ micro USB port to power.

Expansion -40-pin GPIO header.

MIPI DSI to Raspberry Pitouch screen exhibit.

MIPI CSI to Raspberry Pi camera.

Power Supply $-5 \mathrm{~V}$ till $2.4 \mathrm{~A}$ via micro USB port.

Dimensions $-85 \times 56$ × $17 \mathrm{~mm}$.

\section{RESUlt AND Discussion}

The robotized framework suggested through this dissertation is prototyped as well as tried to 2-transport DC arrange. Goals of parts utilized to experiment the model is adequate to transfer generally precise qualities to the IoT stage and the quality of web availability and cell system is sufficient to get warnings immediately when flaws were intentionally made to experiment work of framework. The recurrence in that esteems were transferred to IoT stage additionally assumes main job at the accomplishment of suggested framework.

The framework working is tried utilizing the preliminary 2transport system appeared at figure. Passing issue flows were presented at the same time

Likewise, the framework can catch precise estimations of current in each transport at 1 second interims The alarm given by the framework as appeared in figure was practically immediate, in this manner fulfilling the framework's motivation.
The activity of the framework can further be ad libbed to coordinate modern benchmarks by utilizing

- Current Transformers of appropriate turns proportion rather than Hall-Effect depend current sensors.

- Utilizing IoT stages along maximal paces of information refreshing or restrictive webservers.

- Using Industrially acknowledged Microcontrollers like TI CC 3200

- Utilizing better cell systems and web offices.

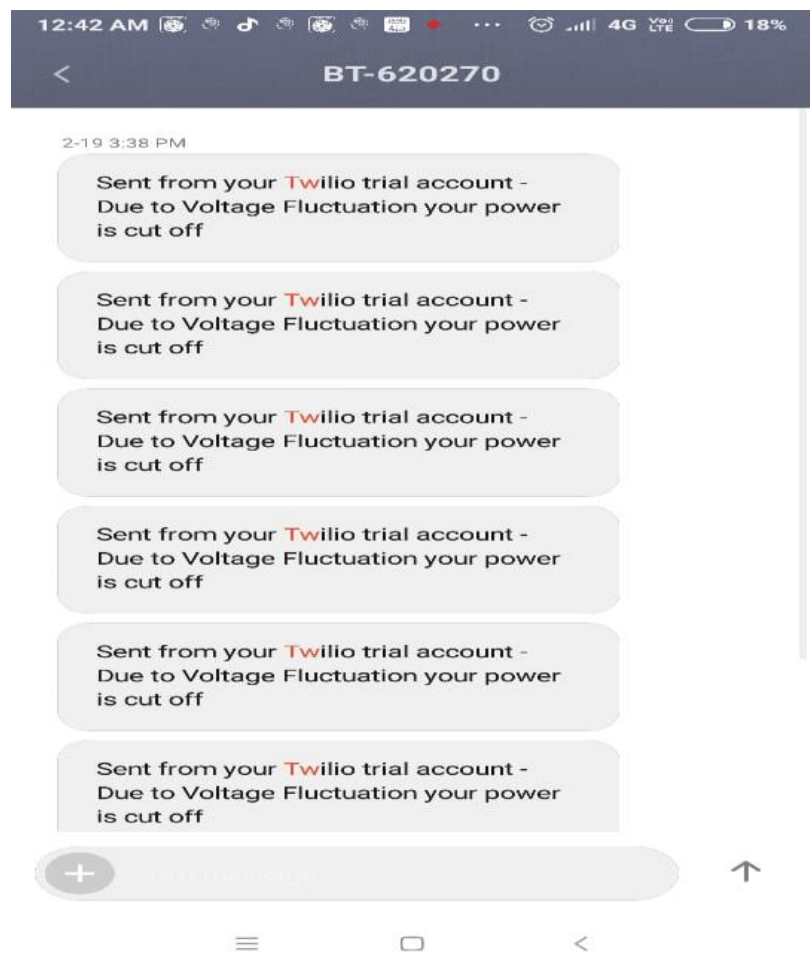

Fig.8. Alerting Notification System

\section{CONCLUSION}

The attention on executing small scale frameworks the nation over to decrease conveyance misfortunes. The vitality segment offers huge extension for development utilizing most recent advancements as well as Automation utilization also IoT at DC miniaturized scale lattices will open the broad scope of chances for enhance the activity of these new age systems. Our task build up a computerized framework dependent on the idea of IoT that always observed the electrical parameters moving through different parts of DC Micro matrices. It effectively recognized and trips the electrical switch and associates the heap to another source. By our task we likewise can anticipate debacles.

\section{REFERENCE}

1. Bakar, A., Ooi, B., Govindasamy, P., Tan, C., Illias, H., and Mokhlis, H. (2014). Directional overcurrent and earth-fault protections for a biomass microgrid 


\section{International Journal of Engineering Applied Sciences and Technology, 2020 \\ Vol. 4, Issue 12, ISSN No. 2455-2143, Pages 506-511 \\ Published Online April 2020 in IJEAST (http://www.ijeast.com)}

system in Malaysia. International Journal of Electrical Power and Energy Systems, 55, 581-591.

2. Bedekar, P., Bhide, S., and Kale, V. (2010). Optimum Coordination of Overcurrent Relay Timing Using Simplex Method. Electric Power Components And Systems, 38(10), 1175-1193.

3. Gnana Swathika, O., and Hemamalini, S. (2016). Prims-Aided Dijkstra Algorithm for Adaptive Protection in Microgrids. IEEE Journal Of Emerging And Selected Topics In Power Electronics, 4(4), 1279-1286.

4. Li, Z., Li, Y., Fu, G., and Li, B. (2012). Directional protection based on fault component energy function in micro-grid. IEEE PES Innovative Smart Grid Technologies. 1-5

5. Majumder, A., Roy, S., and Chowdhuri, S. (2017). Grid-tied VSI protection against grid side faults based on voltage. 2017 IEEE Calcutta Conference (CALCON), (pp. 274-278).

6. Meghwani, A., Srivastava, S., and Chakrabarti, S. (2015). A new protection scheme for DC microgrid using line current derivative. 2015 IEEE Power and Energy Society General Meeting, (pp. 1-5),

7. Mokhberdoran, A., Pirooz Azad, S., Van Hertem, D., Silva, N., and Carvalho, A. (2017). Protection of HVDC Grids Using Unidirectional DC Circuit Breakers and Fast Local Protection Algorithm. 13Th IET International Conference On AC And DC Power Transmission (ACDC 2017), (pp. 11-16).

8. Peralta, V., Faicán, D., and Pinos, J. (2019). Java Program Connection with an Alarm Circuit on Arduino Uno with the Panama Hitek Arduino 2.8 Library. NAWPAY Revista Técnica Tecnológica, 5359.

9. Swathika, O., and Hemamalini, S. (2019). Graph Theory and Optimization Algorithms Aided Adaptive Protection in Reconfigurable Microgrid. Journal of Electrical Engineering and Technology, 15(1), 421431.

10. Swathika, O., Hemamalini, S., Garg, T., and Aggarwal, M. (2016). Hybrid Prims-Johnson's Algorithm for Shortest Path Identification in Reconfigurable Microgrid. Advanced Science Letters, 22(10), 2922-2926.

11. Swathika, O., Hemamalini, S., Mishra, S., Pophali, S., and Barve, N. (2016). Shortest Path Identification in Reconfigurable Microgrid Using Hybrid Bellman Ford-Dijkstra's Algorithm. Advanced Science Letters, 22(10), 2932-2935.
12. Thakkar, A. (2015). Development Of Fingerprint Biometric Attendance Management System Using Wireless Connectivity. International Journal On Recent And Innovation Trends In Computing And Communication, 3(3), 1617-1620.

13. Tu, Q., Li, Y., Liu, W., Huang, M., Zeng, G., Du, B., and Wu, Z. (2018). Arm Overcurrent Protection and Coordination in MMC-HVDC. 2018 IEEE Power and Energy Society General Meeting (PESGM), ( pp 1-5). 